Journal of Al Azhar University Engineering Sector

Vol. 12, No. 45, October, 2017, 1285-1294

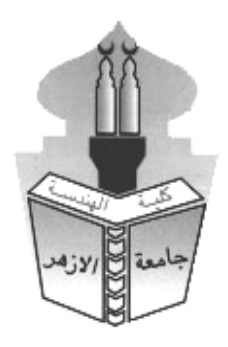

\title{
BRIDGE PYLONS-MODELING AND DESIGN UNDER CFD ANALYSIS
}

\author{
Mohamed O. Elgohary and Walid A.Attia \\ Department of structural engineering, Cairo University
}

\begin{abstract}
Analysis of long span cable bridge such as: Cable stead or Suspension bridges under the effect of fluid structure interaction (FSI) became the main parameter affect design and safety of long spans bridges. In this research we will discussed, the 3D-CFD Finite element modeling of the wind flows around bridges. The 2D-modeling of the bridge deck and the fluid domain around it to get the critical wind speed without flutter occurs, aren't more accurse in the bridge modeling. There are many out of plane parameters that affect the critical wind speed and flutter. But also the 3DModeling of the bridge and the fluid domain using the finite element theory in all of computer programs are very difficult because it need a huge disk space and long time to run the analysis.

Therefore the research studies the world's longest cable-stayed bridge, the Tatara Bridge, was carried out. The design and detailing of the towers, cable alignment, pier and girder shape, and color were studied using scale-models and computer-aided graphics. Because of the scale of this bridge, aesthetic design had to be carried out within severe structural constraints, including aerodynamic stability and economies.
\end{abstract}

\section{INTRODUCTION}

The design of bridges, in particular long spanned ones, is challenging in the sense that there are many complicated issues to be considered. Amidst the loads to be considered, like dead load, live load, wind load, and earthquake load, the wind load becomes the prime concern for the design of the bridges. Traditionally, analysis of a bridge structure and the wind effects are studied using wind tunnel experiments. This usually takes 6-8 weeks and also it is very costly. With the explosive growth in the electronic and computer industry there has been a tremendous increase in the computing power and speed. Therefore, now the shift is towards computer modeling of the wind induced effects on a bridge structure by using the principles of Computational Structural Dynamics (CSD) and Computational Fluid Dynamics (CFD). This reduces cost and time considerably when compared to the traditional approach of wind tunnel experiments for design and analysis of bridges.

The Tacoma Narrows Bridge at Washington, opened in 1940, is a well-known classical example of a bridge failure due to wind. This bridge had abnormally excessive deflections both during construction and service. A wind velocity as low as $42 \mathrm{mph}$ ripped apart the bridge and tore it, buckling the stiffening girders at the mid span (Bowers, 1940). This failure was due to the phenomenon of flutter.

Flutter occurs if the velocity of wind is higher than the critical velocity for a given bridge. This failure brought awareness to the designers around the world that wind can cause aerodynamic instability of bridges resulting in failure. Thus it becomes necessary and important to conduct sufficient aerodynamic studies of the bridge before construction so that the stability of the bridge against wind can be ensured. 


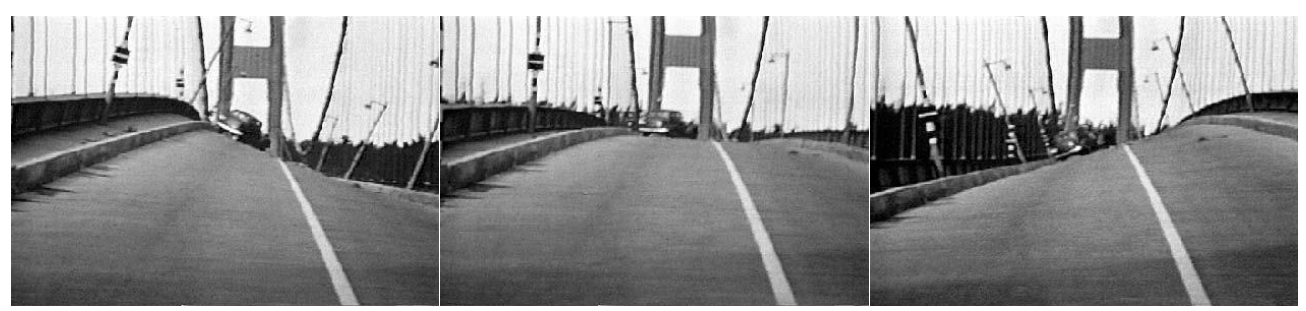

(a)
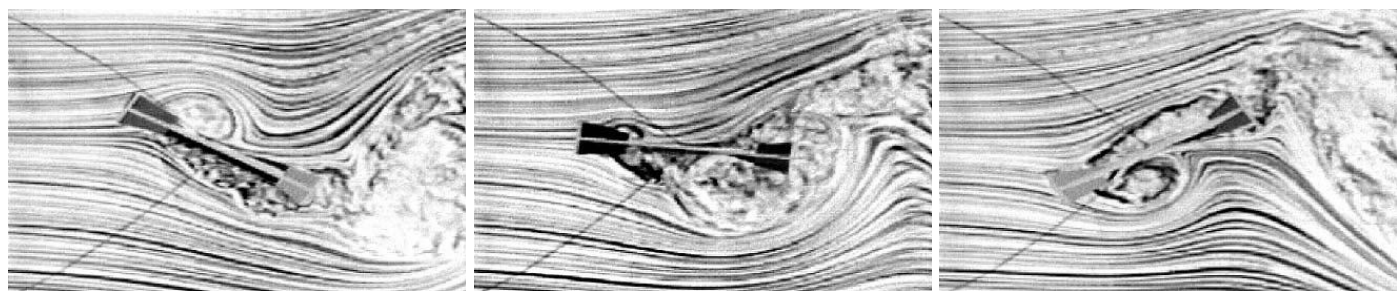

(b)

Fig (1) show The collapse of the Tacoma Narrows Bridge, (a) torsional motion led to collapse, (b) separated vortex descending by flow visualization.

Torsional motion of the bridge superstructure that lead to bridge collapse, and the vertical displacement occurs as shown in Figure (1-1) a, d.

Since this accident, the importance of wind-resistant design for long-span suspension bridges has been highly recognized and has led to many research works and investigations on bridge aerodynamics. Farquharson et al. [2] conducted a series of wind tunnel experiments on the Tacoma Narrows Br. Bleich [3] made analytical studies on the flutter problem. In them they developed the instability analysis using motion-dependent forces on a thin airfoil given by The odorsen [4] in the field of aeronautics.

The Tatara Bridge is the world's longest cable-stayed bridge and has a main span of 890 meters for a total length of 1,480 meters. It forms part of the Nishi-seto Expressway, the westernmost route comprising Japan's Honshu-Shikoku Bridge Project (Fig. 1). The bridge is located in the Seto Inland Sea National Park, so harmony with the surrounding scenery and protection of the environment were crucial issues. When the bridge was first considered in 1973, the plan was to build a Suspension bridge. However, this led to great concern about how to avoid extensive excavations for anchorage $1 \mathrm{~A}$, which was to be constructed on a steep hillside. An overall review of the plan was carried out in 1990, taking into aecount recent developments in stay-cable materials (which have advanced as regards fatigue strength and corrosion protection), computational techniques for multi-degree-of-freedom structures, and construction method. A cable-stayed bridge scheme was proposed as an alternative to the earlier Suspension bridge, and this design was ultimately chosen because it avoided large-scale excavations while also proving more economical and a shorter construction period.

\section{COMPUTATIONAL BRIDGE AERODYNAMICS REVIEW}

The literature also revealed that numerical bridge flutter models usually are $2 \mathrm{D}$ without attempts to include a turbulence model formulation. Other aero elastic phenomena, for example, buffeting [e.g., Turbelin and Gibert (2002)] and vortex-induced oscillation [e.g., Lee et al.(1995) ] have gotten less attention with regard to computational predictions. We note that this does not necessarily imply they are less important.

The Re is about a factor 10 lower compared to the flow near the flutter limit. As with other bluff bodies, capturing three-dimensional (3-D) effects and turbulence in the free-stream and in flow instability zones 
play an important role in replicating these phenomena which in turn make them more difficult to model accurately. Dealing with moderate/high Re naturally raises questions with regard to the importance of modeling three-dimensionality and turbulence. However, accurate flutter predictions appear to be mainly affected by the leading edge separations and the associated pressure forces. Further we note, for sharp leading edges, accurate turbulence modeling and 3-D effects may not be important, whereas it could play a strong role for bridge decks with rounded edges.

One of the first numerical bridge deck simulations relate to the finite-difference method (FDM) and was explored by Fujiwara et al.(1993), both on stationary and moving 2-D grids discredited by 20451 grid points. The Navier-Stokes solutions presented were for Re in the range of $2100-4000$ following wind tunnel experiments. Onset wind-speed predictions agreed in general with the wind tunnel experiments, but discrepancies were found in the amplitudes, these being overestimated by the numerical model. Fujiwara et al .reported that a possible explanation could be the loss of 3-D effects, the 2-D solution predicting larger fluctuations of lift. Furthermore, Onyemelukwe (1993) developed a 2-D finite difference solver on boundary fitted grids. Laminar Navier-Stokes flow solutions were presented for a variety of fixed bridge decks around Re of $1_{\ldots}$ 105: The comprehensive numerical flow visualization studies of Onyemelukwe (1993) were however suppressed by restrictions in CPU and memory. He reported that static force coefficients could not be computed on his IBM 386 PC. It is interesting to note that 10 years after this is no longer a barrier.

Following the wind tunnel tests and in recognition of inadequate turbulence modeling, the Re was physically incorrect $ð 0: 45$ _ $105 \mathrm{P}$ and was kept constant for all wind velocities. The flutter limit was based on 2-D analyses with prescribed deck motions (as opposed to self-excited motions) which was in good agreement with the flutter limit derived from the wind tunnel tests. In contrast to the fully coupled FSI solutions presented in the present simulations, Jenssen and Kvamsdal have a coupling module included in their numerical approach between the FVM fluid solver and an FE structural solver, which resulted in weakly coupled solutions (as described later in Section 3; also shown in Fig.5 ).A background to staggered transient analysis for coupled mechanical system is described in detail by Felippa and Park (1980).Furthermore, Jenssen and Kvamsdal (1999) are currently one of the few researchers who have done numerical bridge studies on 3-D flow models. Their investigations include large eddy simulations on stationary grids expanding a 2-D model of 75000 cells to $40 \_75000$ cells over a distance of $0: 2_{\text {_ }}$ B (where $\mathrm{B}$ is the width of the bridge of $31 \mathrm{~m}$ ) along the span, from which they conclude that the pressure distribution, the steady state lift and pitching moment are in closer agreement with the results of the wind tunnel [see also Jenssen, 1998].However, the present investigations indicate that it does not appear evident that models of this size are required nor that 3-D simulations are required for the flutter limit prediction as 2D coarse models (E1900 nodes in an irregular unstructured grid) without boundary layer modeling seems sufficient, as shown

later in Section 5. Other investigators have also used and developed FVM solvers .For example, De Foy (1998) applied his unsteady incompressible finite volume solver to the Great Belt East bridge deck section. Fluid-only simulations in 2-D were performed for a Re of 1:38 _ 108; assuming laminar flow .De Foy found an St 1/4 0:17 which compares reasonable well with the section model of the wind tunnel (0.11$0.15)$, where Re was in the order of 0:4 105 to $1: 5 \_105$ :

Furthermore, Bruno et al.(2001) applied the FVM which includes a k-e-model when solving the flow past the complicated leading edge detail (including railings) of the Normandy cable-stayed bridge (France).Using parallel computations, Bruno et al.(2001) present 2-D flow results for the stationary bridge deck. Their optimized fluid model consist of an unstructured irregular grid with domain size of 50B 40B: The fluid domain is discredited by 29000 nodes with a cell thickness at the wall of 2 _ 10_3B; where the width of the bridge deck, BE21:2 m: They reported good agreement with the wind tunnel results when comparing force coefficients for the fixed bridge deck. Previous numerical studies using the FE method have been undertaken by, e.g., Lee et al.(1995), Mendes and Branco (1998) and Selvam et al.(2002) . Lee et al.(1995) modeled FSI through moving structured regular grids adopting the ALE formulation. Their 2-D fluid model included a k-e-model and a streamlined-upwind

Petrov Galerkin (SUPG) approximation was assumed. Lee et al.(1995) explored the use of this FE approach on several bridges. The static force coefficients agreed with the results obtained in the wind tunnel but some discrepancies were found with regard to the onset velocity of vortex-induced resonance. 
Mendes and Branco (1998) carried out flow investigations on the Vasco da Gama cable-stayed bridge (Portugal).They assumed laminar flow. However in recognition of the high prototype Re, an incorrect low value of $\operatorname{Re} \partial 3$ _ 103P was used in the demonstration of the flow solutions. For this flow regime, their studies demonstrated that a cross-section with baffles aids the suppression of torsional instability. Furthermore, Selvam (1998) developed an FE model on a rotating moving frame of reference. Their FE model was to the approach bridges of the Great Belt East (Denmark) and, in a more recent analysis, to the main suspension bridge (Selvam et al., 2002).

Selvam et al.present large eddy simulations for an Re in the order of 105: In their recent analysis they use structured regular grids of 14805 nodes contained within a control volume 3B _ 8B: They report on drag coefficients and the flutter instability limit which are in agreement with the wind tunnel tests.

In recent years the spectral method, described by Karniadakis and Sherwin (1999), has made interesting contributions to modelling moving boundaries.A main advantage is resolving the steep boundary layer gradients and shear layers with high-order resolution.Spectral element methods also allow for advecting the flow structures with greater accuracy similar to the vortex methods described below.Using spectral elements also seem to be less CPU demanding compared to the ALE approach. Li et al.(2002) developed and applied their spectral method based on a

rotating moving frame of reference.In a 2-D case study, they applied their model to predict the flutter limit of the Second Forth Road Bridge, UK (Robertson et al., 2003b).

The computational irregular unstructured mesh was made up of 1789 elements and Re ranged between 4167 to 11667 .Good agreement with wind tunnel tests were found although the physical smallscale experiments were based on Re in the order of $1_{-} 105$ to $1_{-} 106$ :

Futhermore, the spectral method of $\mathrm{Li}$ et al.(2002) was also applied to explore the single degree of freedom instability problem of galloping of bluff bodies, e.g.,Robertson et al.(2003a) .Recent investigations provide more detail on bridge deckbehavior, as described by Robertson et al.(2003c) . Grid generation (even in 2-D) can be a time-consuming process, especially when the grids involve bluff bodies. Tothis end, avoiding the use of grids, the discrete-vortex method (DVM) pioneered in the 1960s by Sarpkaya and Chorin as described in the comprehensive reviews [e.g., Sarpkaya (1989), Chorin (1989)], is attractive for FSI analysis.DVM has been popular for many decades (Leonard, 1980).In particular, the method of source panels has been used for studying aerodynamic interactions among various components of an aircraft. The vortex elements are naturally concentrated into areas of nonzero vortices and unlike the grid-based methods, this means that the small-scale flow structures will automatically be captured. However, DVM developers are faced with other difficulties in that several parameters must be prescribed, such as the core radius, defining the maximum circulation to be released for one boundary element and at which distance the surface vortices is to be released from the bridge surface.

The 2-D DVM has been applied to bridge decks by, e.g., Walther (1994), Morgenthal and McRobie (2002), Taylor and Vezza (2002).

In a series of publications, e.g., Walther and Larsen (1997) show flow solutions for fixed bridge decks and flutter limit predictions in agreement with wind tunnel results. Taylor and Vezza (2002) have also developed a DVM solver and present results on stationary and oscillating bridge decks. The derived flutter limit based on prescribed motion compare well with wind tunnel tests.In addition, they show extensive investigations of the flutter motion being suppressed by inclusion of active control vanes. Finally, it should be mentioned that various investigators have used hybrid models.

For example, Brar (1997) developed a coupled finite-difference and vortex-method scheme.An Eulerian finite-difference grid was located in the viscous region next to the bluff body section and the Lagrangian vortex element domain in the flow regions away form the wall boundaries.Flow solutions were presented for $\mathrm{Re}$ of 100-1000.St predictions agreed in general with the solutions of others.In some case, the numerical solution overpredicted the St. This present paper explores the use of a fully coupled FE FSI solver to simulate (1) flow past a fixed deck and (2) to predict the self-excited flutter instability limit.

\section{The Case study}

The case study presented here is the Tatara cable-stayed bridge. The Tatara Bridge is located on the Western Expressway along with eight other bridges and was completed in 1999. The Western 
Expressway goes through nine of the Geiyo Islands with the Tatara Bridge connecting two of these archipelagos together. The Honshü-Shikoku Bridge Authority was set up to connect the largest island in Japan, Honshū with the smallest, and Shikoku which was previously only reached by ferry. The project consists of three expressways - Central, Eastern and Western. Fig.1 shows the perspective of Tatara Bridge.

Tatara Bridge is located in one of the most geologically active parts of the world and designed for some of the world's biggest typhoons prevalent in Japan. In addition, it is located in a very geologically active area and has towithstand major earthquakes. The original proposal for the Tatara Bridge was to be a suspension bridge; however, due to the effect it would have on the surrounding national park area it was changed to a cable stayed bridge. With the main span of $890 \mathrm{~m}$ at the time of construction, it was the longest cablestayed bridge in the world. Tatara Bridge confirmed that a $900 \mathrm{~m}$ span bridge has the load-bearing capacity needed especially under extreme wind loading. Fig. 2 shows the dimensions of the Bridge.

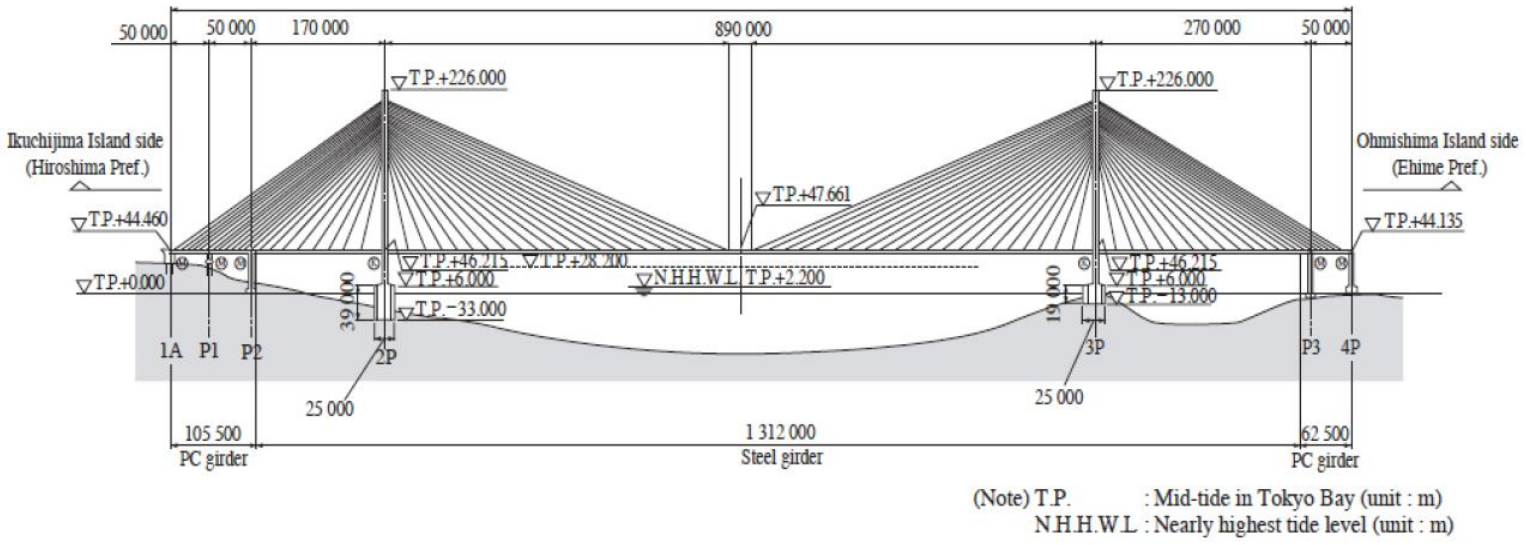

The main tower is $220 \mathrm{~m}$ high and designed as an inverted $\mathrm{Y}$ shape. It has a cross-shaped section with corners cut for higher wind stability and better landscaping. (Material properties, $\mathrm{G}=$ $8.10 \mathrm{E}+09 \mathrm{~kg} / \mathrm{m} 2, \mathrm{E}=2.10 \mathrm{E}+10 \mathrm{~kg} / \mathrm{m} 2, \mathrm{TC}=1.20 \mathrm{E}-05)$. The main girder section, as shown in Fig.3, consists of three spans, $270 \mathrm{~m}, 890 \mathrm{~m}$, and $320 \mathrm{~m}$, and measures $1480 \mathrm{~m}$ in total length. As either side span is shorter than the center span, PC girders are installed at each end of both side span sections as counterweight girders to resist negative reaction. This cable stayed bridge thus uses a steel and PC connection girder. The bridge has a total width of $30.6 \mathrm{~m}$, including a road for motorized bicycles and pedestrians (hereafter called sidewalk) and a girder height of $2.7 \mathrm{~m}$. It uses flat box girders attached with fairings to ensure wind stability. (Prestressed concrete sections properties, $\mathrm{G}=1.22 \mathrm{E}+09 \mathrm{~kg} / \mathrm{m} 2, \mathrm{E}=2.80 \mathrm{E}+06 \mathrm{t} / \mathrm{m} 2, \mathrm{TC}=1.00 \mathrm{E}-05$ and steel sections properties, $\mathrm{G}=8.10 \mathrm{E}+09 \mathrm{~kg} / \mathrm{m} 2, \mathrm{E}=2.10 \mathrm{E}+10 \mathrm{~kg} / \mathrm{m} 2, \mathrm{TC}=1.20 \mathrm{E}-05)$.

\section{Towers}

Towers are the most impressive and symbolic structural elements of a cable-stayed bridge. They extend vertically to a great height, and are clearly to both road users and people Sightseeing. The towers of a cable-stayed bridge are about twice as high as those for an equivalent Suspension bridge because of the way the stay-cables carry the load.

Generally, the towers are determined by basic structural analysis, but where the bridge span is very long, aerodynamics becomes a major problem. Foundation work was already in progress by the time the aesthetic study began, so the design was limited by certain prior constraints, as follows.

1) Size and shape of the foundation

2) Distance between tower columns

3) Tower height

As a result, the towers bend back beneath the deck so as to minimize the size of the foundation. 
Table 1: Comparative study of tower shape

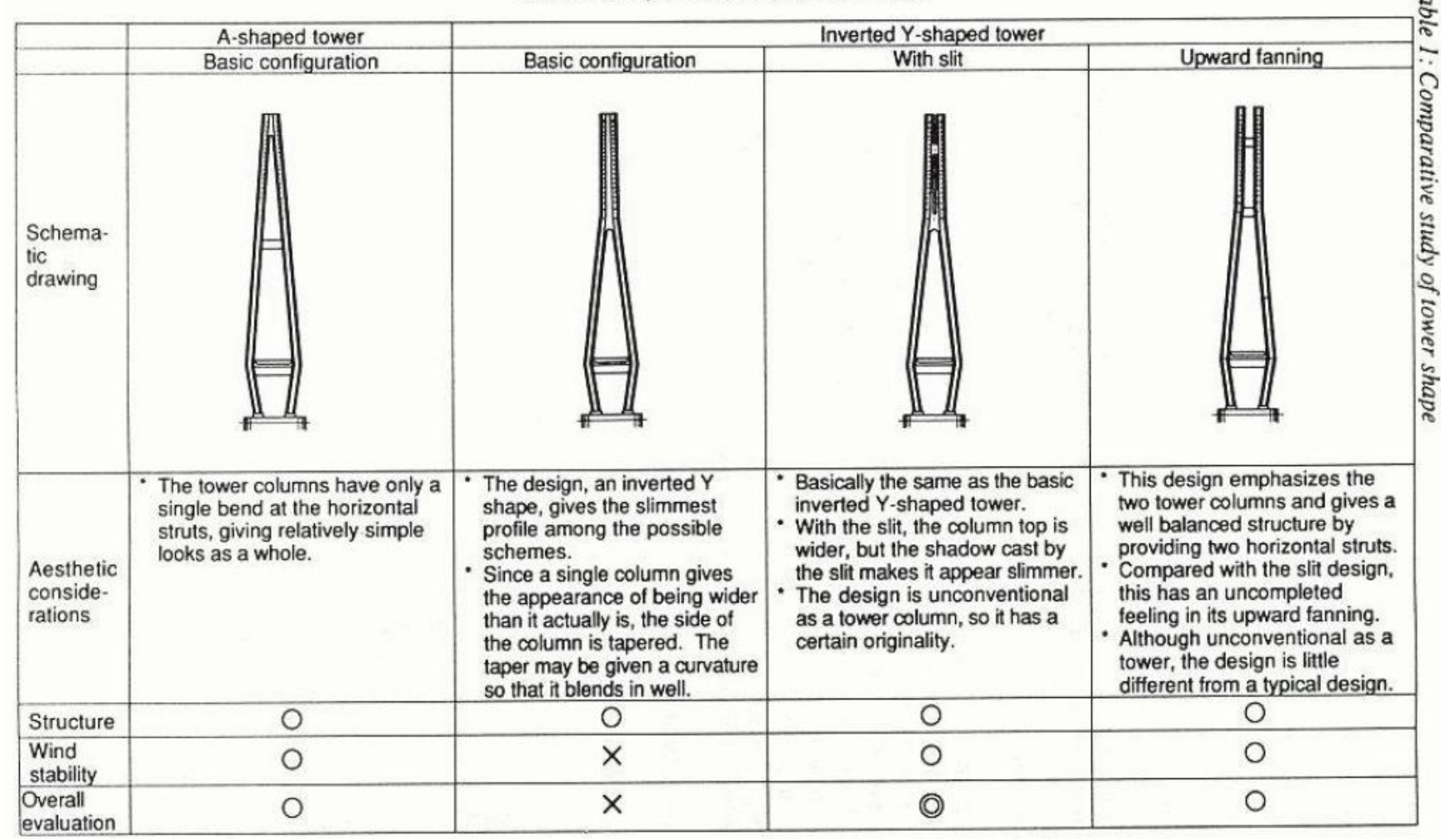

Since the bridge has two cable planes, the possible skeleton designs for the towers are the A-type, inverted Y-type, rigid frame type, and twin column type. Based on these possibilities, four design schemes satisfying the requirements of structure, construction, aerodynamics, and economies were proposed.

Table 1 gives a final comparison of the four schemes and comments on them. The A-type tower offers superior aerodynamic performance, but the inverted $\mathrm{Y}$ design is more appealing if the demands of aerodynamic stability can be met. A full-model wind tunnel test was carried out on an inverted $\mathrm{Y}$ tower to develop a design that would meet the wind stability requirements by adjusting the shape of the column's rectangular section.

Through previous wind tunnel tests, it was known that a rectangular section could be greatly improved as regards aerodynamics by appropriately cutting off the corners. In order to suppress out-of-plane and in-plane vibrations to a sufficient degree, the two faces were given different corner cut-offs (Fig. 2).

The tower columns were bent inward beneath the deck and diagonal members were required between the columns for structural reasons so as to resist lateral earthquake forces. These areas of the design, however, needed some refinement from an aesthetic point of view. Table 2 is a comparative study of five alternative designs for the below-deck shape of the towers. A design with columns wider toward the base was selected.

The base of the tower is expanded, and triangular Spaces are formed above and below the lower horizontal struts, thus together making a diamond shape. This configuration gives prominence to the tower columns, thus creating an uncomplicated, integrated feeling. The expanded bases provide the impression of stability. As regards tower detailing, the following features were incorporated in the final design

(Fig. 3): 
Table 2 Comparative study of below-deck tower shape

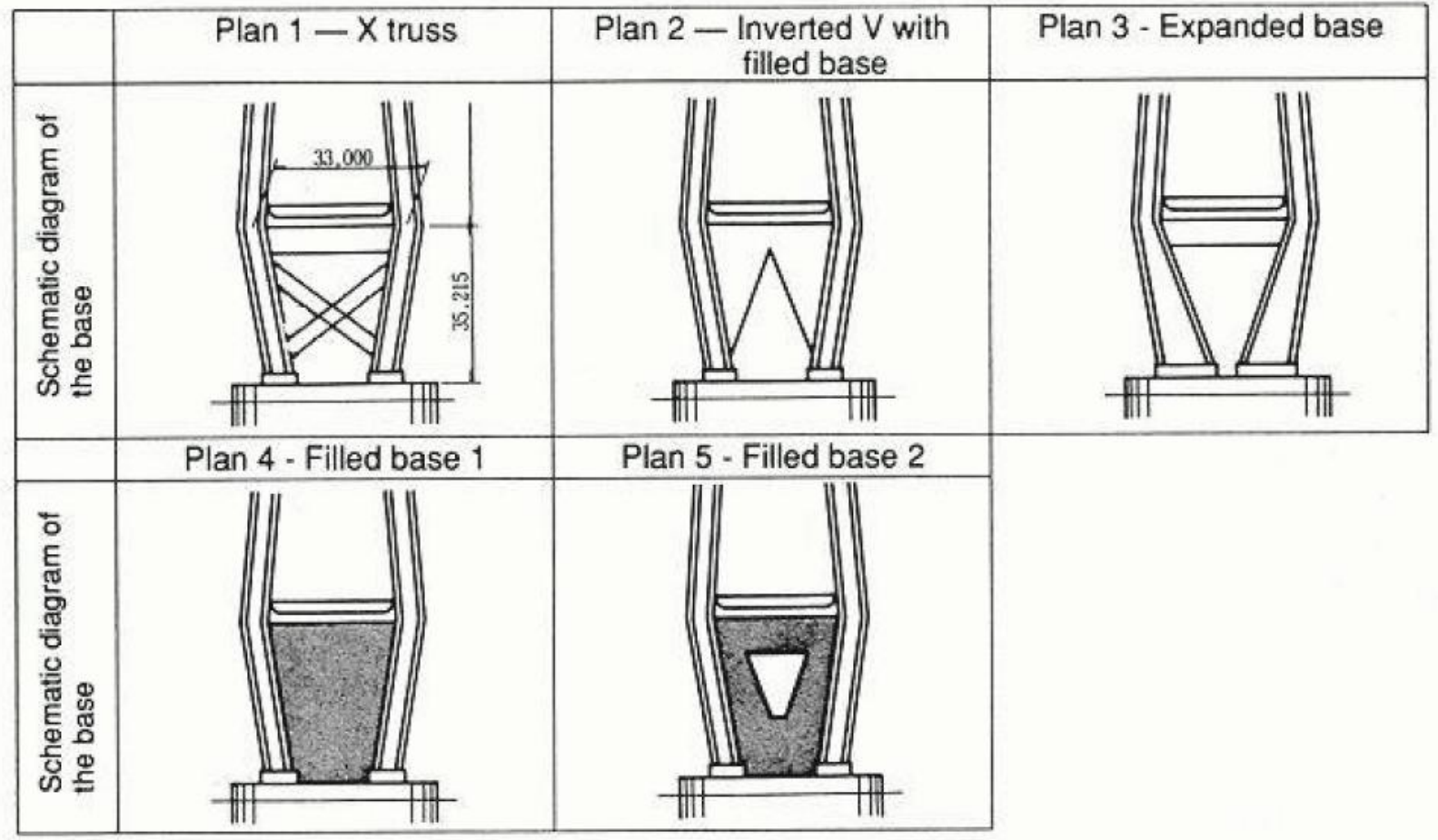

(1) The tower tops were slanted to emphasize the feeling of soaring into the sky.

(2) Strut-column connections were smoothed into arcs so as to preserve the soaring feeling.

(3) A knuckle point was added to the column to match the fairing tip of the girder, thus maintaining continuity of the girder at the towers.

Piers and abutments also match the shape of the towers.

\section{COLOR}

Superstructure color was studied and a light gray (Munsell No. N7.5) was selected for the towers and girders for the following reasons:

(1) It matches with the concept of "light and shade".

(2) It matches with surrounding scenery of a string of small islands with evergreen Vegetation.

(3) It provides uniformity with the other ten long-span bridges planned or already built as part of this route.

(4) It naturally matches the color of the prestressed concrete girders.

(5) It fades less, as proved by twenty years of experience around the bridge site. The stay-cables are covered with Polyethylene piping which has carbon content for weather durability. Although coloring technology is available for this type of cables, it was decided to add no color since black was expected to fit into the environment, and further, colored cable looks black anyway against a bright sky and is also expensive.

\section{The 3D-Fluid Domain models \&parameters affect this analysis}

In this case the governing equations of motion are the incompressible isothermal NSEs described (gravity term here excluded) by: Reynolds averaging, the solution variables in the instantaneous (exact) Naier-Stokes equations are in three direction velocity $\mathrm{X}, \mathrm{Y}$, and $\mathrm{Z}$.

The dependent variables in this case are the pressure $\mathrm{p}$; and the fluid particle velocity vector $\mathbf{u}=\{\mathbf{u}, \mathbf{v}, \mathbf{w}\}^{T}$ with reference to $\mathrm{x} ; \mathrm{y}$ and $\mathrm{z}$ Cartesian directions, where $\mathrm{i} ; \mathrm{j}$ and $\mathrm{k}$ are unit vectors. We assume a constant air density 
$\gamma=1.25 \frac{\mathrm{kg}^{\mathbf{3}}}{\mathrm{m}}$.The kinematic viscosity $\mathbf{v}\left(=\frac{\boldsymbol{\mu}}{\boldsymbol{\rho}}\right.$, where $\boldsymbol{\mu}$ is the dylamic viscosity $)$ is assumed constant with

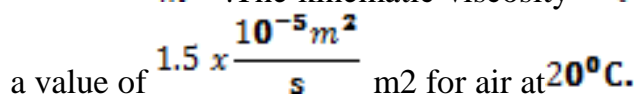

The figures (3) show a complete 3D-Domain this study will take in the considerations some parameters that have been neglect in the 2D-Domain study as:

1- The pylon stiffness and height.

2- The bridge main span and secondary span

3- The bridge cable effect in the analysis

4- The wind speed and motion all-around the bridge not in one side.

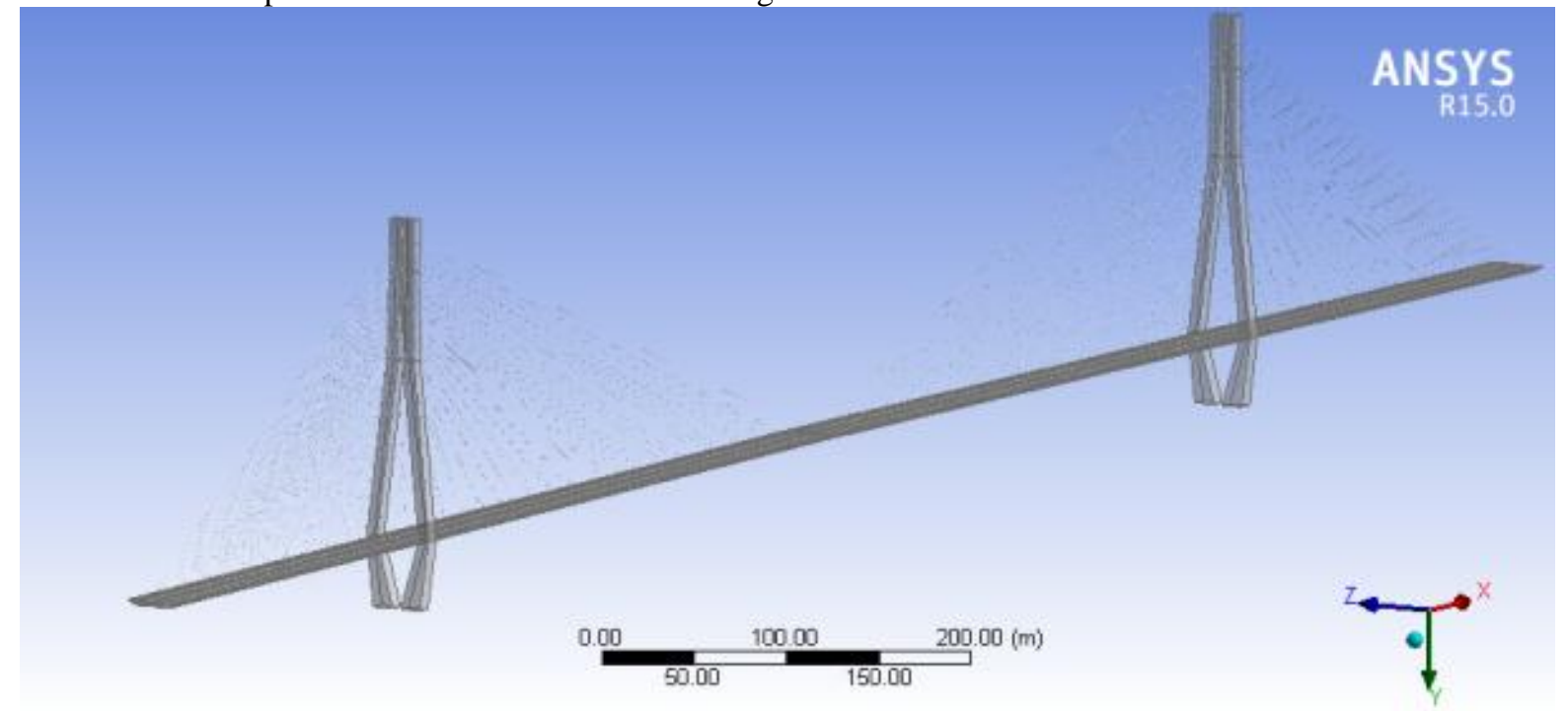

Figure (3) show the general view of the bridge that we are study

\section{RESULTS}

After this study we consider a three important shaped of the wind 3D-Domain around the bridge. Using a complete 3D-bridge with a complete domain is difficult and has long time and large space in computer analysis. Thus we conclude using a half bridge model with a half 3D-Doamin shape or rectangle and making a symmetric cutting wall with symmetric boundary condition and initial condition.

In the case studies presented in the following section, prior to the full fluid-structure analyses of moving bridge decks , a series of simulations are undertaken to observe the unsteady flow patterns around a stationary structure in an attempt to establish mesh-modeling criteria for the flow phenomena of interest. The solution of the fluid equations results in computed pressures and tractions which act on the bridge. The resulting forces on the bridge deck are then computed by integrating the pressures and friction components along the boundary on the deck surfaces. The net forces (per unit span) are usually expressed in a dimensionless form, and referred to as the aerodynamic coefficients, the drag $C_{D}(t)=\frac{F_{D}(t)}{0.5 \rho U^{2} D}$; the lift $C_{L}(t)=\frac{F_{L}(t)}{0.5 \rho U^{2} B} \quad$ and the moment $\quad C_{M}(t)=\frac{M(t)}{0.5 \rho U^{2} B^{2}}$ 


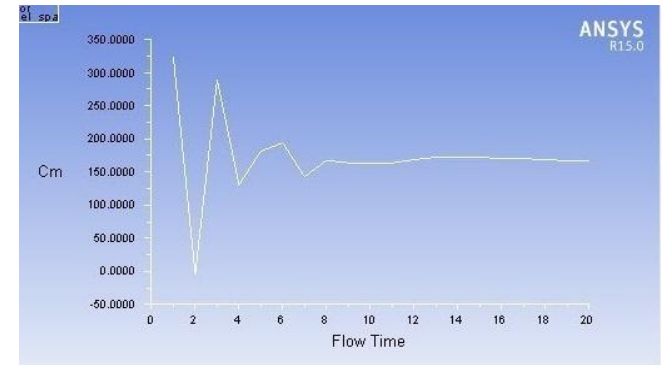

(a)

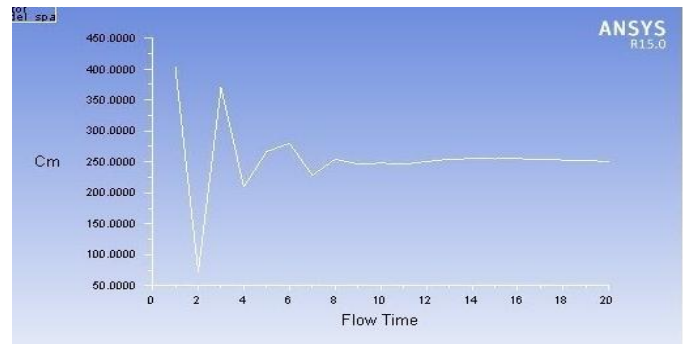

(b)

Figure (7-a) show the momentum coefficients from 3D analysis, (7-b) A half 3D model with half Domain.

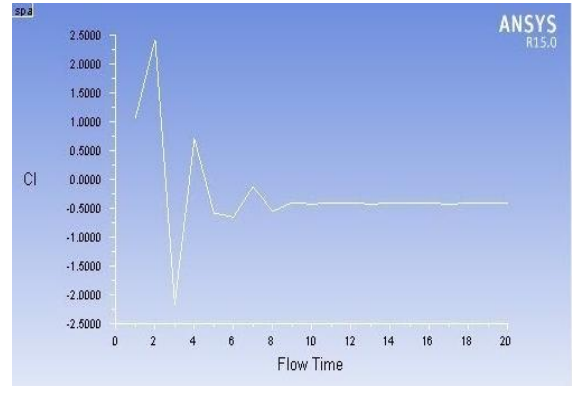

(a)

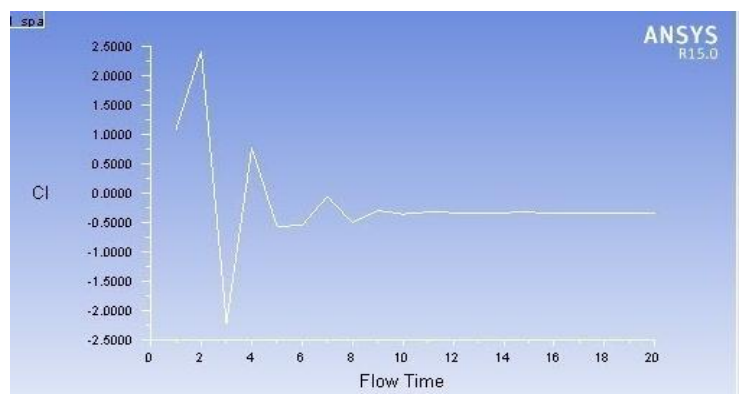

(b)

Figure (8-a) show the left coefficients from 3D analysis, (8-b) A half 3D model with half Domain.

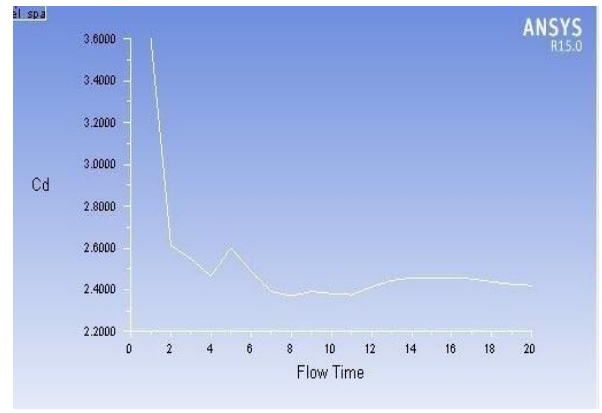

(a)

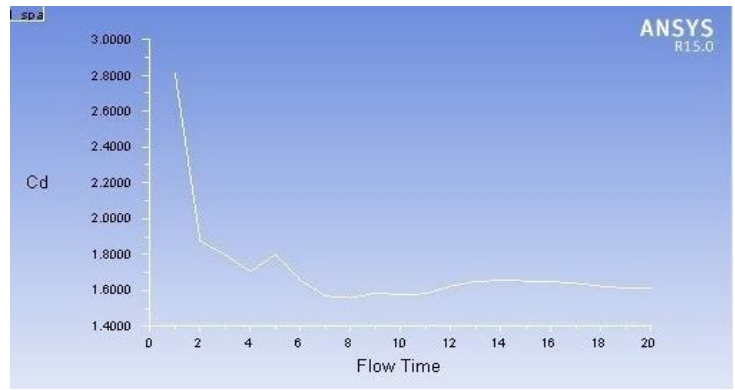

(b)

Figure (9-a) show the Drag coefficients from 3D analysis, (9-b) A half 3D model with half Domain.

\section{CONCLUSION}

This study of aesthetics made it possible to develop a more balanced design. The final design is shown in Photo 1.

Although many structural elements of this design were determined by structural requirements, including aerodynamics, it was still possible through careful consideration to come up with a balanced, meaningful design without increasing costs. The use of scale-models and computeraided photo-montages was very helpful in carrying out comparative studies.

Long-span bridges require extensive wind tunnel testing. The objective of this research is to determine the 3D-Airodynamic model that make the computer model is very similar to the expensive physical model tests that are currently required to determine the aerodynamic crosssection of a single structure. This study describes the use of a coupled fluid-structure interaction (FSI) finite element (FE) solver. Aerodynamic effects of flutter-bridge motion are investigated using fluid and structural two-dimension FEs on moving non adaptive grids. The moving interface between fluid and structure is modeled through the arbitrary Lagrangian-Eulerian (ALE) formulation. 
First, simulation of fluid flow around a fixed bridge deck was simulated. The FE model was validated using different meshes for a range of high Reynolds numbers. Time-domain vortex-shedding analysis was presented. Some questions as to model accuracy were raised due to a discrepancy in Strouhal number compared to physical experiments. The reason being that the turbulent flow around the fixed deck problem was not captured reliably with the present viscous FE solver. However, it was shown, that the mechanisms of flutter are independent of the much higher-frequency bluff body shedding phenomenon and was therefore not explored any further in this study. Second, flutter simulations were presented showing the ability of the FE model to self-excite into flexural-torsional flutter. The FE simulations showed that, when the flutter limit is reached, the structure controls the fluid flow. The flutter limit of the long-span bridge deck was found to be in good agreement with the wind tunnel results and other numerical methods. Furthermore, it is also recognized that the flat-plate flutter theory of Theodore (1935) gives comparatively accurate solutions despite the assumption of in viscid flow, suggesting that accurate modeling of the boundary layer may not be so critical for this aero elastic phenomenon. The models used, despite the laminar flow assumption and the coarseness of the meshes compared to those of other investigators, may be close to what is required for this type of flow simulation, that is about 1900 nodes in an irregular unstructured grid. However, a higher CPU requirement, but not necessarily parallel processing, than those used here is desirable if the FE ALE approach is to prove to be a viable supplementary tool to the wind tunnel. In conclusion, prediction of flutter instability for sharp edge bridge decks does not appear sensitive to turbulence and three-dimensional flow structure modeling.

\section{Acknowledgement}

I am very grateful to Prof.Dr.Walid Abd elateif, Department of structural engineering, Cairo University for the many help and advices that he gives to me.

\section{REFERENCES}

1. (1)-Anju, A., Maruoka, A., Kawahara, M., 1997. 2-D fluid-structure interaction problems by an arbitrary Lagrangian )Eulerian finite element method.International Journal of Computational Fluid Dynamics 8, 1-9.

2. (2)Ansys Inc., 1999. Spectrum Solver (Version 2.0) Command Reference and Theory Manual. Ansys Inc., USA (Spectrum wascommercially available in 1993-1999 and is a registered trademark of Ansys Inc.).

3. (3)Brar, P.S., 1997. Numerical calculation of bluff body flutter derivatives via computational fluid dynamics. Ph.D. Thesis, The John Hopkins University, Baltimore, MD, USA.

4. (4)Bruno, L., Khris, S., Marcillat, J., 2001. Numerical simulation of the effect of section details and partial streamlining on the aerodynamics of bridge decks.Journal of Wind and Structures 4, 315-332.

5. (5)Chorin, A.J., 1989. Numerical study of slightly viscous flow. Journal of Fluid Mechanics 57(4), 785-796. 1973.

6. (6)Dahle, L.A., Reed, K., Aarsnes, J.V., 1990. Model tests with submerged floating tube bridges. In: Krokeborg, J. (Ed.), Proceedings of the Second Symposium on Strait Crossings.Balkema, Trondheim, Norway.

7. (7)De Foy, B., 1998. Unsteady incompressible flow solver with optimised operators. Ph.D. Thesis, Cambridge University, Department of Engineering, Cambridge, England.

8. (8)DMI and SINTEF, 1993a.Wind-tun nel tests. Storebælt East Bridge.Suspensio n bridge.Section model tests, II. Technical Report 92063.00.01, Danish Maritime Institute, Lyngby, Denmark (Used with permission from Storebælt A/S).

9. (9)DMI and SINTEF, 1993b.Wind-tunnel tests.Storebælt East Bridge.Tender evaluation, suspension bridge. Alternative sections.

10. (10)Section model tests, I. Technical Report 91023-10.00. Revision 0., Danish Maritime Institute, Lyngby, Denmark (Used with permission from Storebælt A/S). Donea, J., Fasolistella, P., Giuliani, S., 1977. Lagrangian and Eulerian finite element techniques for transient fluid-structure interaction problems. Transactio ns Fourth SMIRT Conference, San Fransisco, 15-19 August 1977, USA (Paper B1/2). 